\title{
A study of liver disorder and its consequences in pregnant women with jaundice in tertiary care centre in Belgaum, Karnataka, India
}

\author{
Dr. Divyakala Karegoudar ${ }^{1}$, Dr. Patel Ronilkumar Dhirubhai ${ }^{2}$, Mukesh Dhital ${ }^{2}$, \\ *Dr. Kapil Amgain ${ }^{2}$ \\ Jawaharlal Nehru Medical College, KLE University, Belgaum-590010, India \\ ${ }^{I}$ Assistant Professor, Department of Obstetrics and Gynaecology \\ ${ }^{2}$ Post graduate student, Jawaharlal Nehru Medical College
}

\begin{abstract}
Pregnancy with jaundice is regarded as high risk pregnancy so it is considered very important sign during antenatal check up. Incidence of jaundice in pregnancy is 0.4-0.9/1000 in India. It complicates 3-5\% of pregnancies and is one of the important causes of maternal and neonatal morbidity and mortality worldwide. The normal physiological and hormonal profiles may alter throughout the pregnancy. The present retrospective study was carried out in the Department of Obstetrics and Gynecology, KLE's Dr. Prabhakar Kore Hospital and research centre from September 2011 to August 2012 to determine the causative factors responsible for jaundice in pregnant women and to assess its consequences in pregnancy. During this retrospective study of 3 years, 9,612 deliveries were conducted. Among them 9,167 were live birth and 445 were stillbirth. Overall $37(0.4 \%)$ women had clinical and biochemical evidences of jaundice. Their mean age was $24.78 \pm 3.66$ years and the mean period of gestation ( $34.7 \pm 3.42$ weeks). Among them predominant cause of jaundice was HELLP 24(64.86\%) followed by acute viral hepatitis and Leptospirosis 5(13.51\%) of each. Severe PIH was the most common co-morbid conditions observed in 20(54.05\%) of the pregnant women. And the commonest cause of maternal mortality 15(53.57\%) was HELLP followed by severe anaemia 3(10.75\%). Among 37 births, 53.85\% were live births, $28.21 \%$ were fresh still births and $17.95 \%$ were macerated still births. Among the $53.85 \%$ of live births, foetal mortality was seen in $4.76 \%$. Pregnancy with liver disorder results in very high foetal as well as maternal morbidity and mortality. HELLP was found to be the predominant cause of Jaundice and associated mortality in pregnancy. It might cause mild to severe complication in pregnancy. Pregnancy with liver disorder results in high foetal as well as maternal morbidity and mortality. Thus early diagnosis and proper management is essential to reduce the high maternal mortality in pregnant women with jaundice.
\end{abstract}

Key Words: Hepatitis, maternal mortality, foetal mortality, pregnancy, HELLP, Jaundice, Pre-eclampsia

\section{Introduction}

Pregnancy with jaundice is considered as high risk pregnancy. It is an important sign encountered during clinical examination. It complicates 3-5\% of pregnancies and is one of the important causes of maternal and neonatal morbidity and mortality worldwide ${ }^{[1]}$. Throughout the pregnancy there exists alteration in normal physiological and hormonal profiles. Among them liver disorders comprise up to $3 \%$ of all pregnancy complications ${ }^{[2]}$. Incidence of jaundice in pregnancy is $0.4-0.9 / 1000$ in India ${ }^{[3]}$.

Jaundice in pregnancy can be classified into three categories. i) Jaundice unique to pregnancy such as cholestasis of pregnancy, preeclampsia ii) Jaundice exacerbated by Pregnancy including viral hepatitis like Hepatitis E, Hepatitis B infection of Herpes Simplex virus during pregnancy which may lead to fulminant hepatic failure iii) Jaundice due to Pre-existing liver disorders like chronic active hepatitis and wilson's diseases. Viral hepatitis is the most frequent cause of jaundice associated with pregnant woman. Incidence of hepatitis varies markedly throughout the world.

In developed countries the incidence is around $0.1 \%$ whereas in developing countries it can range from $3-20 \%$ or higher. There is no difference in the course of the disease in pregnant and non pregnant women in developed countries. However the incidence of maternal morbidity and mortality presented with fulminant hepatitis is higher in developing countries ${ }^{[4]}$.

\section{"Corresponding Author:}

Dr. Kapil Amgain, Jawaharlal Nehru Medical College, KLE University, Belgaum-590010, India

Email: dr.kapilamgain@gmail.com, drdivyajk@gmail.com 
HEV and HBV infections were most frequent cause of fulminant hepatic failure in pregnancy ${ }^{[3,4,5]}$. As far as developing countries like India is concerned, Hepatitis E is the most common cause of fulminant hepatic failure. Fulminant hepatitis was seen in high percentage in third trimester pregnant women with high maternal mortality ranging from $15-45 \%{ }^{[6,7]}$. Pruritus with elevated serum bile acids which is often seen in second half of pregnancy resolving after delivery is considered as intrahepatic cholestasis ${ }^{[8]}$. Recurrence in subsequent pregnancies is widely seen. The incidence which is seen in Europe ranges from $0.1 \%$ to $1.5 \%$ of pregnancies compared with a much significant incidence in Scandinavia ${ }^{[9]}$. Maternal morbidity is low and therefore the importance of this disorder is related to its effects on the fetus. Intrahepatic cholestasis can lead to chronic placental insufficiency, resulting in fetal complications that include anoxia, prematurity, perinatal death, fetal distress, and stillbirth. Various studies have been done to evaluate the maternal and foetal outcome in different conditions causing jaundice in pregnancy. However the prevalence and aetiology of jaundice still remain debatable in developing and developed countries. Hence the present study was an attempt to determine the causative factors responsible for jaundice in pregnant women.

\section{Methodology}

This is a retrospective study conducted in the Department of Obstetrics and Gynaecology, KLE Dr Prabhakar Kore Hospital and Medical Research Centre Belgaum, between September 2011 to August 2012. All pregnant women with jaundice based on clinical and biochemical evidences from 2009 to 2012 were included in this study. Ethical clearance was taken from the institutional ethics committee of Jawaharlal Nehru medical college Belgaum. All relevant data were collected, scrutinized for the clinical and biochemical profile, etiology, maternal outcome and foetal outcome. The data obtained were tabulated and analysed by using appropriate statistical method on Microsoft excel and SPSS version 20. The results were presented in tables, Pie charts, histogram as well as in the narrative form as per necessity.

\section{Result}

Pregnant women with jaundice based on clinical and biochemical evidences were studied from January 2009 to January 2012. During this period 9,612 deliveries were conducted (live birth=9,167 and still birth=445) out of which $37(0.4 \%)$ pregnant women had clinical as well as biochemical evidence of jaundice. Out of 37 pregnant women with jaundice, majority of them $28(75.68 \%$ ) belongs to age group of 21 to 30 years and $7(18.92 \%)$ were of age group less than 20 years. Only 2(5.41\%) were above 30 years. Others results were tabulated as well as shown in the diagrams as follows.

Table 1: Cause of Jaundice in pregnant women

\begin{tabular}{|l|l|l|l|}
\hline S.N. & Cause of Jaundice & Number & Percentage \\
\hline 1 & HELLP & 24 & $64.86 \%$ \\
\hline 3 & Acute viral hepatitis & 5 & $13.52 \%$ \\
\hline 4 & Leptospirosis & 5 & $13.52 \%$ \\
\hline 5 & Gilbert Syndrome & 1 & $2.7 \%$ \\
\hline 6. & Hepatitis B & 1 & $2.7 \%$ \\
\hline 7 & Cholestasis jaundice & 1 & $2.7 \%$ \\
\hline Total & $\mathbf{3 7}$ & $\mathbf{1 0 0 \%}$ & $100 \%$ \\
\hline
\end{tabular}

Figure No. 1: Mode of delivery

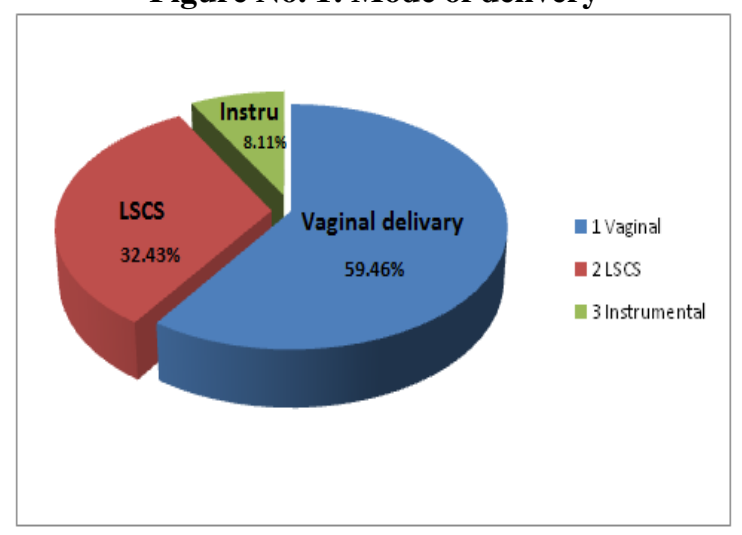


Table No.2: Outcome of jaundice in the pregnant women

\begin{tabular}{|l|l|l|l|}
\hline S.N. & Mode of delivery & Frequency & Percentage \\
\hline 1 & Improved & 9 & $24.32 \%$ \\
\hline 2 & Mortality & 28 & $75.68 \%$ \\
\hline Total & & $\mathbf{3 7}$ & $\mathbf{1 0 0 \%}$ \\
\hline
\end{tabular}

Table No. 3: Cause of mortality

\begin{tabular}{|l|l|l|l|}
\hline S.N. & Cause of Mortality & Frequency & Percentage \\
\hline 1 & HELLP & 15 & $53.57 \%$ \\
\hline 2 & Severe anemia & 3 & $10.75 \%$ \\
\hline 3 & Pulmonary embolism & 2 & $7.14 \%$ \\
\hline 4 & Abruptio Placenta & 2 & $7.14 \%$ \\
\hline 5 & Septicaemia & 1 & $7.14 \%$ \\
\hline 6 & Multiorgan dusfuncton sundrome & 1 & $3.57 \%$ \\
\hline 7 & Leptospirosis & 1 & $3.57 \%$ \\
\hline 8 & Cerebral Malaria & 1 & $3.57 \%$ \\
\hline 9 & ARDP & 1 & $3.57 \%$ \\
\hline & Total & 28 & $100 \%$ \\
\hline
\end{tabular}

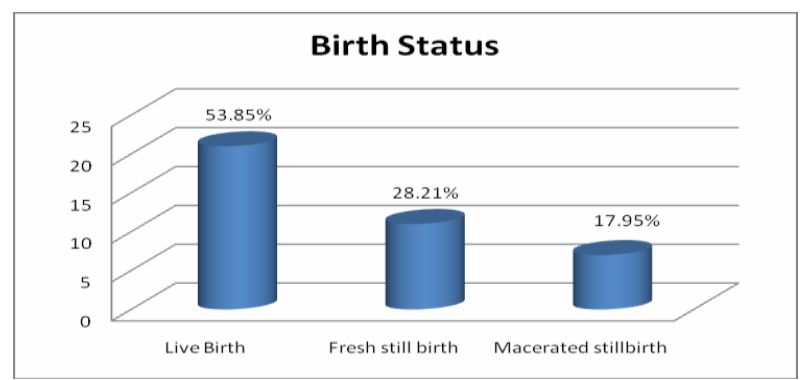

Figure No. 2: Birth status of baby delivered from jaundiced mothers

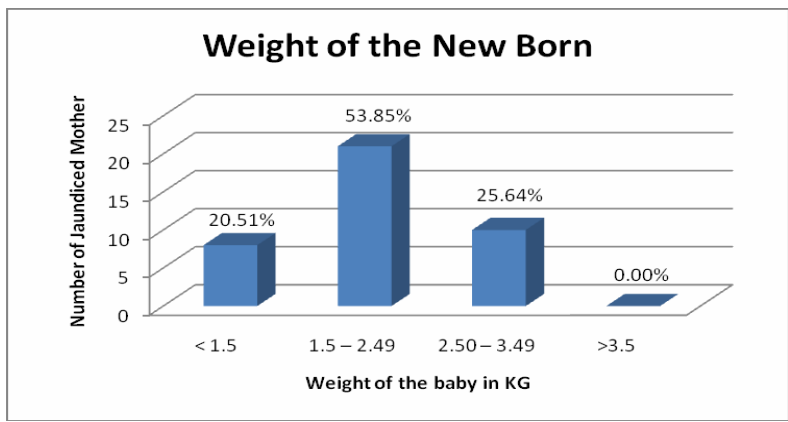

Figure No. 3: Weight of the baby delivered from the jaundiced mother

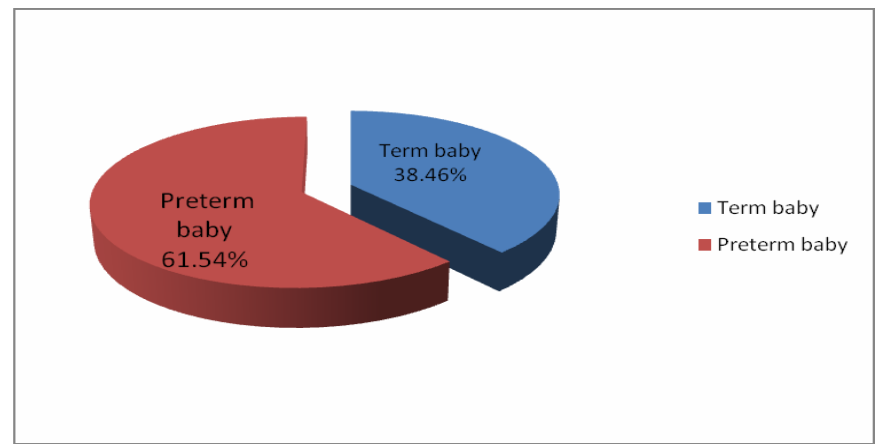

Figure No. 4: Foetal outcome of the jaundiced mothers 
Table 4: Requirement of NICU for the baby delivered from jaundiced mothers

\begin{tabular}{|l|l|l|l|}
\hline SN & Requirement of NICU & Frequency & Percentage \\
\hline 1 & Required & 5 & 13.51 \\
\hline 2 & Not required & 32 & 86.49 \\
\hline Total & 37 & 100 \\
\hline
\end{tabular}

Table No 5. Bilirubin Level in pregnant women

\begin{tabular}{|l|l|l|l|}
\hline SN & Serum Bilirubin $(\mathbf{m g} \%)$ & Frequency & Percentage \\
\hline 1 & $<10$ & 25 & $67.57 \%$ \\
\hline 2 & $10.0-14.90$ & 4 & 10.815 \\
\hline 3 & $15.0-19.9$ & 5 & $13.51 \%$ \\
\hline 4 & $>20$ & 3 & $8.11 \%$ \\
\hline Total & & 37 & 100 \\
\hline
\end{tabular}

Table No 6: Co-morbid conditions in pregnant women

\begin{tabular}{|l|l|l|l|}
\hline SN & Co-morbid conditions & Frequency & Percentage \\
\hline 1 & Abruptio placenta & 1 & $2.7 \%$ \\
\hline 2 & Acute Renal Failure & 1 & $2.7 \%$ \\
\hline 3 & Cerebral Malaria & 2 & $5.41 \%$ \\
\hline 4 & IUGR & 1 & $8.11 \%$ \\
\hline 5 & Sever PIH & 20 & $54.05 \%$ \\
\hline
\end{tabular}

\section{Discussion}

In the present study, 9,612 deliveries were conducted from 2009 to 2012 . Out of them 9,167 were live birth and 445 were still births. Overall, 37(0.4\%) women had clinical and biochemical evidences of jaundice. Similar findings were reported in a retrospective study ${ }^{[10]}$ from Mumbai among 7080 pregnant women where they found 48 women $(0.65 \%)$ with jaundice. In another prospective study ${ }^{[11]}$ at Mumbai, pregnant women with liver diseases were evaluated and followed up till two weeks postpartum or death. Liver disease was found in $107(0.9 \%)$ of 12,061 pregnancies. Comparing to these studies our study revealed less percentage of pregnant women with jaundice. In the present study, most of the women 28(75.68\%) belonged to age group of 21 to 30 years. Mean age was $24.78 \pm 3.66$ years. Similar study done in 7,280 pregnant women reported with mean age 26 years which is comparable to the present study ${ }^{[3]}$. Almost half $18(48.65 \%)$ were primi gravida followed by $13(35.14 \%)$ which were parity status one. Of the 37 cases more than half $21(56.76 \%)$ were not registered for ANC check up.

HELLP is commonly encountered in the second or third trimester but can also arise after delivery. Advanced maternal age, multi-parity, and white ethnic origin are the major risk factors for the development of HELLP ${ }^{[12]}$. In the present study, the predominant cause of jaundice was found to be of HELLP in 24(64.86\%) of women followed by, acute viral hepatitis and Leptospirosis $5(13.51 \%)$ of each. Infant mortality rate of 6-7\% in perinatal period has been reported primarily as a consequence of prematurity, or secondary to maternal complications ${ }^{[13]}$. Hepatic disorders in pregnancy are not common, but have clinical importance because of its serious adverse effects on both mother and fetus. Liver disease in pregnancy can be the cause of subtle changes in liver biochemical profile or can be presented with fulminant hepatic failure. These disorders are notorious and might need to be handled by clinically experienced physicians in tertiary care centre. The maternal and foetal survival rate has improved because of better understanding of the pathogenesis of these disorders and higher standards of clinical care ${ }^{[12]}$. Gallstones are often seen in pregnancy because of hyper cholesterol secretion in the second and third trimester, increased lithogenicity of the bile, and reduced gallbladder motility ${ }^{[14]}$. About $10 \%$ of pregnant women encounter either gallstones or viscous biliary sludge ${ }^{[15]}$. But in present study none of the pregnant women had gall stone.

Few cases of viral hepatitis $6(16.21 \%)$ were found among them only one case $(2.7 \%)$ was presented with Hepatitis B virus. Hepatitis virus infection in pregnancy has a similar type of clinical course as compared to that in the non-pregnant population ${ }^{[16]}$. A recent study has declared the presence of ascites and hypertension as factors useful to differentiate liver disease specific to pregnancy from viral hepatitis ${ }^{[17]}$. Increased severity of disease is found to be associated with advanced maternal age, with severe infection in the third trimester associated with an increased risk of prematurity ${ }^{[18]}$. 


\section{Conclusion}

Pregnancy with liver disorder results in very high foetal as well as maternal morbidity and mortality. HELLP was found to be the predominant cause of Jaundice and associated mortality in pregnancy. It might cause mild to severe complication in pregnancy. Pregnancy with liver disorder results in high foetal as well as maternal morbidity and mortality. Thus early diagnosis and proper management is essential to reduce the high maternal mortality in jaundiced pregnant women.

\section{References}

[1]. R.K. Jain. Management of jaundice in pregnancy. Medicine update 2010, 20, 470-6

[2]. C.L. Ch'ng, M. Morgan, I. Hainsworth J.G. Kingham. Prospective study of liver dysfunction in pregnancy in Southwest Wales.Gut 2002; 51: 876-80.

[3]. T. Nagaria, S. Agrawal, Fetomaternal outcome in jaundice during pregnancy, J Obstet Gynecol India, 2005, 55(5), 424-7.

[4]. M.S. Khuroo, S. Kamili, Aetiology and prognostic factors in acute liver failure in india, J viral Hepat., 2003, 10, 224 -31

[5]. Y.B. Yang, X.M. Li, Z.M. Shi, L. Ma, Pregnant women with fulminent hepatic failure caused by hepatitis B virus infection: A case report, World J Gastroenterol, 2004, 10 (15), 2305-6.

[6]. M.S. Khuroo, Hepatitis E: The enterically transmitted Non-A, Non-B Hepatitis, J Gastroenterol 1991, 10, 96-100.

[7]. M.S. Khuroo, S. Kamili, S. Jameel, Vertical transmission of Hepatitis E Virus, Lancet, 1995, 345, 1025-6.

[8]. Reyes H. The enigma of intrahepatic cholestasis of pregnancy: lessons from Chile. Hepatology 1982; 2: 87-96.

[9]. G. Schneider, T.C. Paus, G.A. Kullak-Ublick, Linkage between a new splicing site mutation in the MDR3 alias ABCB4 gene and intrahepatic cholestasis of pregnancy. Hepatology 2007; 45: 150-58

[10]. C.L Yi, N. Barge, A.R. Dalal, Study of Jaundice in Pregnancy in a tertiary care institute in india, Bombay Hospital J, 2011, 53, 569.

[11]. U. Rathi, M. Bapat, P. Rathi, P. Abraham, Effect of liver disease in maternal and foetal outcome - A prospective study. Indian J Gastroenterol, 2007, 26;61

[12]. Deepak Joshi, Andra James, Alberto Quaglia, Rachel H Westbrook, Michael A Heneghan, Liver disease in pregnancy, Lancet 2010; 375: 594-605

[13]. D. Mihu, N. Costin, C.M. Mihu, A. Seicean, R. Ciortea. HELLP syndrome - a multisystemic disorder. J Gastrointestin Liver Dis 2007; 16: 419-24.

[14]. G. Lindseth, M.Y. Bird-Baker. Risk factors for cholelithiasis in pregnancy. Res Nurs Health 2004; $27: 382-91$.

[15]. C.W. Ko, S.A. Beresford, S.J. Schulte, A.M. Matsumoto, S.P. Lee. Incidence, natural history, and risk factors for biliary sludge and stones during pregnancy. Hepatology 2005; 41: 359-65.

[16]. E. Elinav, I.Z. Ben-Dov, Y. Shapira. Acute hepatitis A infection in pregnancy is associated with high rates of gestational complications and preterm labor. Gastroenterology 2006; 130: 1129-34.

[17]. H. Devarbhavi, W.K. Kremers, R. Dierkhising, L. Padmanabhan. Pregnancy-associated acute liver disease and acute viral hepatitis: diff erentiation, course and outcome. J Hepatol 2008; 49: 930-35.

[18]. I.R. Willner, M.D. Uhl, S.C Howard, E.Q. Williams, C.A. Riely, B. Waters. Serious hepatitis A: an analysis of patients hospitalized during an urban epidemic in the United States. Ann Intern Med 1998; 128: 111-14. 